\title{
O Papel da Ecocardiografia sob Estresse na Detecção Precoce de Disfunção Diastólica em Pacientes com Doença Pulmonar Obstrutiva Crônica Não Grave
}

\author{
The Role of Stress Echocardiography in the Early Detection of Diastolic Dysfunction in Non-Severe Chronic \\ Obstructive Pulmonary Disease Patients
}

\author{
Zheyna Cherneva ${ }^{1}$ e Radostina Cherneva ${ }^{2}$ (1) \\ Medical Institute of the Ministry of Internal Affairs, ${ }^{1}$ Sofia - Bulgária \\ Saint Sophia University Hospital of Pulmonary Diseases, ${ }^{2}$ Sofia - Bulgária
}

\section{Resumo}

Fundamento: A dispneia por esforço é uma queixa comum de pacientes com insuficiência cardíaca com fração de ejeção preservada (ICFEP) e doença pulmonar obstrutiva crônica (DPOC). A ICFEP é comum na DPOC e é um fator de risco independente para a progressão e exacerbação da doença. A detecção precoce, portanto, tem grande relevância clínica.

Objetivos: O objetivo deste estudo foi detectar a frequência de ICFEP mascarada em pacientes com DPOC não grave com dispneia aos esforços, sem doença cardiovascular manifesta, e analisar a correlação entre ICFEP mascarada e os parâmetros do teste cardiopulmonar de exercício (TCPE).

Métodos: Aplicamos o TCPE em 104 pacientes com DPOC não grave com dispneia aos esforços, sem doença cardiovascular evidente. A ecocardiografia foi realizada antes e no pico do TCPE. Os valores de corte para disfunção diastólica ventricular esquerda e direita induzida por estresse (DDVE/DDVD) foram E/e' $>15$; E/e $\mathbf{e}^{\prime}>6$, respectivamente. A análise de correlação foi feita entre os parâmetros do TCPE e o estresse E/d'. Valor de $p<0,05$ foi considerado significativo.

Resultados: 64\% dos pacientes tinham DDVE induzida por estresse; 78\% tinham DDVD induzida por estresse. Ambos os grupos com estresse DDVE e DDVD obtiveram carga menor, $\mathrm{V}^{\prime} \mathrm{O}_{2}$ e pulso de $\mathrm{O}_{2}$ mais baixos, além de apresentarem redução na eficiência ventilatória (maiores inclinações de $\mathrm{VE} / \mathrm{VCO}_{2}$ ). Nenhum dos parâmetros do TCPE foram correlacionados com E/e' DDVE/DDVD induzida por estresse.

Conclusão: Há uma alta prevalência de disfunção diastólica induzida por estresse em pacientes com DPOC não grave com dispneia aos esforços, sem doença cardiovascular evidente. Nenhum dos parâmetros do TCPE se correlaciona com E/e' induzida por estresse. Isso demanda a realização de Ecocardiografia sob estresse por exercício (EES) e TCPE para detecção precoce e manejo adequado da ICFEP mascarada nesta população. (Arq Bras Cardiol. 2021; 116(2):259-265)

Palavras-chave: Ecocardiografia sob Estresse/métodos; Insuficiência Cardíaca Diastólica; Volume Sistólico; Doença Pulmonar Obstrutiva Crônica; Testes de Função Respiratória.

\footnotetext{
Abstract

Background: Exertional dyspnea is a common complaint of patients with heart failure with preserved ejection fraction (HFpEF) and chronic obstructive pulmonary disease (COPD). HFpEF is common in COPD and is an independent risk factor for disease progression and exacerbation. Early detection, therefore, has great clinical relevance.

Objectives: The aim of the study is to detect the frequency of masked HFpEF in non-severe COPD patients with exertional dyspnea, free of overt cardiovascular disease, and to analyze the correlation between masked HFpEF and the cardiopulmonary exercise testing (CPET) parameters.

Methods: We applied the CPET in 104 non-severe COPD patients with exertional dyspnea, free of overt cardiovascular disease. Echocardiography was performed before and at peak CPET. Cut-off values for stress-induced left and right ventricular diastolic dysfunction $(L V D D / R V D D)$ were E/e'> 15; E/e' $>6$, respectively. Correlation analysis was done between CPET parameters and stress E/e'. A p-value $<0.05$ was considered significant.
}

Correspondência: Zheyna Cherneva •

Medical Institute of the Ministry of Internal Affairs - Cardiology - bul General Skobelev 78 Sofia 1000 - Bulgária

E-mail: jenicherneva@yahoo.com

Artigo recebido em 15/07/2019, revisado em 24/11/2019, aceito em 22/01/2020

DOI: https://doi.org/10.36660/abc.20190623 
Results: $64 \%$ of the patients had stress-induced LVDD; 78\% had stress-induced RVDD. Both groups with stress LVDD and RVDD achieved lower load, lower $\mathrm{V}^{\prime} \mathrm{O}_{2}$ and $\mathrm{O}_{2}$-pulse, besides showing reduced ventilatory efficiency (higher VE/VCO slopes). None of the CPET parameters were correlated to stress-induced left or right E/e'.

Conclusion: There is a high prevalence of stress-induced diastolic dysfunction in non-severe COPD patients with exertional dyspnea, free of overt cardiovascular disease. None of the CPET parameters correlates to stress-induced E/e'. This demands the performance of Exercise stress echocardiography (ESE) and CPET for the early detection and proper management of masked HFpEF in this population. (Arq Bras Cardiol. 2021; 116(2):259-265)

Keywords: Echocardiography,Stress/methods; Heart Failure,Diastolic; Stroke Volume; Pulmonary Disease,Chronic Obstructive; Respiratory Funstion Tests

Full texts in English - http://www.arquivosonline.com.br

\section{Introdução}

As anormalidades cardiovasculares são comuns na doença pulmonar obstrutiva crônica (DPOC) ${ }^{1,2}$ A rigidez arterial está presente mesmo em pacientes com DPOC leve e sem doenças cardiovasculares. Trata-se de um fator de risco cardiovascular independente que contribui para o desenvolvimento de disfunção diastólica. ${ }^{3}$ Dispneia e intolerância ao exercício são sintomas comuns da DPOC e disfunção diastólica. ${ }^{4}$ Estudos recentes com grandes coortes de pacientes identificaram um fenótipo cardiovascular em pacientes com DPOC que apresentam curso clínico e prognóstico diferentes. ${ }^{5}$ O diagnóstico e o manejo precoces são, portanto, muito importantes do ponto de vista clínico.

O teste cardiopulmonar de exercício (TCPE) pode distinguir dispneia cardíaca e respiratória ou diminuição da atividade física. ${ }^{6-9}$ A combinação de ecocardiografia sob estresse por exercício (EES) e TCPE é uma abordagem confiável para identificar pacientes com insuficiência cardíaca mascarada pela fração de ejeção preservada (ICFEP). Além disso, os resultados das medições invasivas são comparáveis aos dados obtidos em estudos não invasivos durante o EES. ${ }^{10}$

Os objetivos de nosso estudo foram: 1) detectar a frequência de disfunção diastólica subclínica do ventrículo esquerdo (VE) e do ventrículo direito (VD) em pacientes com DPOC não grave sem doença cardiovascular; 2) estabelecer uma correlação entre exercício cardiopulmonar e os parâmetros ecocardiográficos para disfunção diastólica (E/e').

\section{Materiais e métodos}

\section{Pacientes e protocolo de estudo}

Este foi um estudo retrospectivo realizado com 224 pacientes ambulatoriais clinicamente estáveis com diagnóstico de DPOC no Hospital Universitário de Doenças Respiratórias "St. Sophia", Sofia. Apenas 163 deles atenderam aos critérios de inclusão para DPOC não grave: volume expiratório forçado no primeiro segundo maior que $50 \%\left(\mathrm{VEF}_{1}>50 \%\right)$. Todos os indivíduos apresentavam dispneia aos esforços, mas um total de 104 pacientes ( 64 homens, 40 mulheres; idade média de $62,9 \pm 7,5$ anos) foram considerados elegíveis, assumindo-se os critérios de exclusão. O período de recrutamento foi entre maio de 2017 e abril de 2018, e foi aprovado pelo Comitê de Ética local (protocolo 5/12.03.2018). Todos os participantes assinaram o termo de consentimento livre e esclarecido antes do início do estudo.
Foram considerados os seguintes critérios de exclusão: 1) fração de ejeção do ventrículo esquerdo (FEVE) $<50 \%$; 2) disfunção diastólica do ventrículo esquerdo em repouso superior ao primeiro grau; 3) achados ecocardiográficos sugerindo hipertensão pulmonar (pressão arterial pulmonar sistólica $>36 \mathrm{mmHg}$, regurgitação tricúspide (RT), velocidade máxima do jato $>2,8 \mathrm{~m} / \mathrm{s}$; 4) cardiopatia valvar; 5) cardiomiopatia documentada; 6) hipertensão grave não controlada (pressão arterial sistólica $>180 \mathrm{mmHg}$ e pressão arterial diastólica $>90 \mathrm{mmHg}$ ); 7) fibrilação atrial ou arritmia ventricular maligna; 8) doença isquêmica do coração; 9) anemia; 10) diabetes mellitus; 11) câncer; 12) doença renal crônica (DRC); 13) cirurgia torácica ou abdominal recente; 14) exacerbação recente (nos últimos três meses); 15) mudança recente (nos últimos três meses) na terapia médica.

\section{Procedimentos}

\section{Teste de função pulmonar}

Todos os indivíduos foram submetidos a um exame clínico preliminar, incluindo radiografia de tórax, espirometria, eletrocardiografia e ecocardiografia. Os pacientes elegíveis para o estudo realizaram espirometria e teste ergométrico em Vyntus, teste cardiopulmonar de exercício (Carefusion, Alemanha), de acordo com as diretrizes da European Respiratory Society (ERS). ${ }^{11}$ Apenas pacientes com obstrução leve a moderada das vias aéreas $\left(\mathrm{VEF}_{1}>50 \%\right)$ foram selecionados.

\section{Protocolo de teste de estresse - teste cardiopulmonar de exercício (TCPE)}

Um protocolo em rampa contínua foi aplicado de acordo com diretrizes. ${ }^{6}$ Após dois minutos de pedalada sem carga (fase de repouso $0 \mathrm{~W}$ ), seguiu-se uma fase de aquecimento de três minutos (20W). A fase de teste incluiu incrementos de carga de $20 \mathrm{~W} / 2 \mathrm{~min}$. Os pacientes foram orientados a pedalar a 60 rotações por minuto.

Os gases expiratórios foram coletados respiração a respiração. O pico de $\mathrm{VO}_{2}$ foi expresso como o maior valor médio de 30 segundos, obtido na última etapa do teste de esforço. Os valores de pico de $\mathrm{VO}_{2}$ são expressos como $-\mathrm{O}_{2}$ $\mathrm{ml} / \mathrm{kg} / \mathrm{min}$. A eficiência ventilatória ( $\mathrm{EV} / \mathrm{VCO}_{2}$ ) foi medida pelo método $\mathrm{V}$-slope. O pico da razão de troca respiratória (pRTR) foi o maior valor médio de 30 segundos no último estágio do teste. RTR $>1,10$ ao final do teste EES-TCPE foi considerado como esforço máximo. 


\section{Métodos ecocardiográficos}

Foram realizados ecocardiografia modo $\mathrm{M}$, bidimensional com Doppler. ${ }^{12,13}$ Incidências apicais de quatro câmaras foram usadas para medir os volumes das câmaras com base na regra modificada de Simpson e a fração de ejeção do VE foi considerada preservada se $>50 \%$. A análise da ecocardiografia Doppler tecidual foi realizada na dimensão septo-lateral do anel mitral e na dimensão lateral do anel tricúspide para avaliar as ondas sistólica $(S)$ e diastólica miocárdica $\left(E^{\prime}, A^{\prime}\right)$ do VE e VD. O valor $E^{\prime}$ foi usado como média das medições mediais e laterais. $\mathrm{O}$ pico da razão $\mathrm{E} / \mathrm{e}^{\prime}>15$ foi considerado um marcador para disfunção diastólica ventricular esquerda induzida por estresse.

A função sistólica do ventrículo direito foi avaliada por meio da excursão sistólica do plano do anel tricúspide (TAPSE) e da velocidade de pico $S$ do Doppler tecidual. A espessura da parede do ventrículo direito (EPVD) foi medida a partir do corte subcostal no eixo longo na extremidade do folheto tricúspide anterior ao final da diástole. A pressão pulmonar foi calculada diretamente pela amostra da insuficiência tricúspide e indiretamente pelo tempo de aceleração (TA) do fluxo pulmonar. $\mathrm{O}$ índice de volume do átrio direito (IVAD) foi medido com o volume sistólico final do ventrículo direito pela regra modificada de Simpson. A disfunção diastólica do VD induzida por estresse foi considerada se a razão E/e' induzida por estresse fosse $>6$. Todos os parâmetros foram medidos no final da expiração e três vezes durante diferentes ciclos cardíacos. ${ }^{14}$

\section{Análise estatística}

Os dados demográficos e clínicos foram apresentados em estatística descritiva. O teste de Kolmogorov-Smirnov foi usado para explorar a normalidade da distribuição. As variáveis contínuas em cada grupo foram expressas como mediana e intervalo interquartil quando os dados não foram normalmente distribuídos, e como média \pm desvio-padrão (DP) se a distribuição foi normal. Variáveis categóricas foram apresentadas em proporções. Os dados foram comparados entre pacientes com e sem DDVE, bem como entre pacientes com e sem DDVD. O teste t de Student não pareado foi usado para analisar variáveis contínuas normalmente distribuídas. O teste de Mann-Whitney-U foi usado em outros casos. Variáveis categóricas foram comparadas pelo teste de $\chi^{2}$ ou exato de Fisher. A correlação de Spearman foi usada para avaliar a associação entre os parâmetros TCPE e a razão E/e' induzida por estresse nos ventrículos esquerdo e direito.

Em todos os casos, um valor de p inferior a 0,05 foi considerado significativo, conforme determinado pelas estatísticas do software SPSS ${ }^{\circledR} 13.0$ (SPSS, Inc, Chicago, Ill).

\section{Resultados}

No grupo DPOC, 30\% (32/104) dos pacientes apresentaram DDVE grau I em repouso; 14\% (15/104) tinham DDVD grau I em repouso e apenas $3 \%(4 / 104)$ tinham tanto DDVE quanto DDVD em repouso. Após o TCPE, a ecocardiografia sob estresse estabeleceu que 64\% (67/104) dos indivíduos tinham DDVE induzida por estresse e 78\% (82/104) tinham DDVD induzida por estresse. Todos os pacientes com DDVE induzida por estresse também tinham DDVD induzida por estresse. Os dados demográficos e clínicos dos pacientes estão listados na Tabela 1. Os parâmetros ecocardiográficos dos pacientes estão apresentados na Tabela 2. Exceto para IVAD, EPVD, TA e pressão arterial pulmonar sistólica após carga, nenhuma outra diferença significativa foi encontrada entre os pacientes com e sem DDVE (Tabelas 1 e 2). Os resultados para pacientes com e sem DDVD foram semelhantes (Tabelas 1 e 2).

A capacidade de exercício foi reduzida em pacientes com DPOC com disfunção diastólica direita e esquerda induzida por estresse, em comparação com pacientes sem (Tabela 1). Os pacientes DPOC-DDVD/DDVE alcançaram carga mais baixa, $\mathrm{VO}_{2}$ e pulso de $\mathrm{O}_{2}$ mais baixos. Tiveram um desempenho com inclinações de VM/ $\mathrm{VCO}_{2}$ significativamente maiores (Tabela 1). Nenhum dos parâmetros TCPE foi associado à razão E/e' esquerda ou direita induzida por estresse (Tabela 3.)

\section{Discussão}

Nossos principais achados foram: 1) $64 \%$ dos pacientes com DPOC não grave e dispneia aos esforços que estão livres de doença cardiovascular clinicamente evidente têm DDVE induzida por estresse; 1) $78 \%$ do mesmo grupo de pacientes têm DDVD induzida por estresse; 3) nenhum dos parâmetros TCPE foi correlacionado com a razão $\mathrm{E} / \mathrm{e}^{\prime}$ induzida por estresse no ventrículo esquerdo ou direito. Até onde sabemos, este é o primeiro estudo usando a combinação EES-TCPE em pacientes com DPOC não grave, dispneia aos esforços e livres de doenças cardiovasculares evidentes. Aumento da razão E/e' induzida por estresse $>15$ do ventrículo esquerdo foi detectado em $64 \%$ deles; elevação induzida por estresse da razão $\mathrm{E} / \mathrm{e}^{\prime}>6$ no ventrículo direito foi encontrada em $78 \%$ dos casos. Não podemos comparar nossos dados com outros estudos de populações de pacientes com DPOC não grave porque a maioria deles relata a incidência de disfunção diastólica em repouso. ${ }^{15-17}$

Nedeljkovic et al. realizaram EES em uma população de 87 pacientes hipertensos com dispneia aos esforços e função ventricular esquerda normal. Reportaram, em 9,2\% dos pacientes, razão por estresse $\mathrm{E} / \mathrm{e}^{\prime}>15 .{ }^{18}$ Kaiser et al. também investigaram uma população geral de 87 pacientes com dispneia aos esforços e relataram disfunção diastólica em $9 \%$ deles. ${ }^{19}$

A maior prevalência de disfunção diastólica de estresse que descrevemos em pacientes com DPOC confirma que a própria DPOC é um fator de risco cardiovascular. ${ }^{20,21}$ Rigidez arterial é uma característica da DPOC, independentemente do impacto do tabagismo. O estresse da parede ventricular observado durante a respiração também é relatado como um mecanismo fisiopatológico independente para a remodelação do VE em pacientes com DPOC leve sem patologia cardiovascular evidente. ${ }^{22}$ Tanto a rigidez arterial quanto o estresse da parede ventricular causam fibrose ventricular difusa em pacientes com DPOC livres de doenças cardiovasculares. ${ }^{23,24}$

Em nosso estudo, pacientes com disfunção diastólica induzida por estresse (ambos DDVE e DDVD) atingem carga, $\mathrm{VO}_{2}$ e pulso de $\mathrm{O}_{2}$ mais baixos e apresentam desempenho em inclinações de $\mathrm{VM} / \mathrm{VCO}_{2}$ significativamente maiores. 


\begin{tabular}{|c|c|c|c|c|}
\hline & $\begin{array}{l}\text { Pacientes sem DDVE por } \\
\text { estresse (37) }\end{array}$ & $\begin{array}{l}\text { Pacientes com DDVE por } \\
\text { estresse (67) }\end{array}$ & $\begin{array}{c}\text { Pacientes sem DDVD por } \\
\text { estresse (22) }\end{array}$ & $\begin{array}{c}\text { Pacientes com DDVD por } \\
\text { estresse (82) }\end{array}$ \\
\hline \multicolumn{5}{|l|}{ Dados demográficos } \\
\hline Idade, ano & $60,44 \pm 7,72$ & $64,16 \pm 6,97^{*}$ & $6,95 \pm 7,36$ & $63,74 \pm 7,60^{*}$ \\
\hline Masculino:feminino, $n$ & 21:16 & $44: 23^{\ddagger}$ & $14: 8$ & $50: 32^{\ddagger}$ \\
\hline Pacote, anos & $27,21(23,87-31,76)$ & $33,79(30,51 \pm 37,87)^{\dagger}$ & $26,52(23,46-30,43)$ & $32,11(28,82-36,13)^{\ddagger}$ \\
\hline Índice de massa corpórea, $\mathrm{kg} / \mathrm{m}^{2}$ & $27,00(24,75-31,00)$ & $27,96(22,75-30,75)^{\dagger}$ & $28,00(25,25-30,5)$ & $26,52(22,72-30,61)^{\dagger}$ \\
\hline \multicolumn{5}{|l|}{ Função respiratória } \\
\hline $\mathrm{CVF}, \mathrm{l} / \mathrm{min}$ & $2,06(1,76-3,09)$ & $2,34(1,77-3,09)^{\dagger}$ & $2,05(2,11-3,73)$ & $2,21(1,71-2,93)^{\dagger}$ \\
\hline $\mathrm{FEV}_{1}, 1 / \mathrm{min}$ & $1,31(0,94-1,53)$ & $1,36(1,14-1,75)^{\dagger}$ & $1,60(1,15-2,42)$ & $1,52(1,14-1,75)^{\dagger}$ \\
\hline $\mathrm{FEV}_{1} / \mathrm{CVF} \%$ & $60,5(46,91-67,47)$ & $53,30(45,76-66,55)^{\dagger}$ & $65,50(54,81-68,82)$ & $62,59(46,57-66,79)^{\dagger}$ \\
\hline \multicolumn{5}{|l|}{ Equilíbrio ácido-base } \\
\hline $\mathrm{pO}_{2}, \mathrm{mmHg}$ & $68,60(63,4-71,8)$ & $71,35(64,7-74)^{\dagger}$ & $67,20(63,56-71,68)$ & $70,6(63,2-74)^{\dagger}$ \\
\hline $\mathrm{pCO}_{2}, \mathrm{mmHg}$ & $32,30(30,1-35,37)$ & $37,65(32,5-40)^{\dagger}$ & $34,73(31,27-39,21)$ & $35,7(32,5-40)^{\dagger}$ \\
\hline Saturação, \% & $94,9(94,4-95,25)$ & $95,00(94,02-95,67)^{\dagger}$ & $94,75(92,67-95,0)$ & $95,00(93,9-95,5)^{\dagger}$ \\
\hline \multicolumn{5}{|l|}{ Parâmetros TCPE } \\
\hline Pico de carga, W & $82,75(69,8-89,1)$ & - $76,05(68,4-92,1)^{\dagger}$ & $86,66(78,65-94,76)$ & $\boldsymbol{- \cdot 7 3 , 0 8}(68,93-83,16)^{\dagger}$ \\
\hline Pico de VM, I/min & $40(34-52,5)$ & $38,50(32-48)^{\dagger}$ & $41,1(32,12-48,17)$ & $39,07(31,89-48,32)^{\dagger}$ \\
\hline Pico de V'O $\mathrm{O}_{2}, \mathrm{ml} / \mathrm{min} / \mathrm{kg}$ & $14,30(12,6-16,15)$ & $13,90(12,67-15,7)^{\dagger}$ & $14,30(12,6-16,15)$ & $13,40(15,77-12,55)^{\dagger}$ \\
\hline RTR & $1,06(0,98-1,19)$ & $1,09(1,00-1,28)^{\dagger}$ & $1,05(0,98-1,18)$ & $1,08(1,01-1,19)^{\dagger}$ \\
\hline Pico de pulso de $\mathrm{O}_{2} \mathrm{ml} / \mathrm{min} / \mathrm{kg}$ & $9,80(9,5-12,2)$ & $\cdot 7,90(6,15-9,32)^{\dagger}$ & $9,51(9,02-13,1)$ & 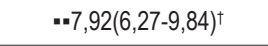 \\
\hline Pico de inclinação VM/NCO & $34,08(33,98-36,72)$ & -36,93 $(34,19-38,74)^{\dagger}$ & $34,11(33,78-36,89)$ & $\mathbf{- 3 6 , 9 8}(34,26-38,91)^{\dagger}$ \\
\hline
\end{tabular}

*Teste t não pareado; †Teste U de Mann-Whitney; Teste ¥chi-quadrado; DDVE: disfunção diastólica do ventrículo esquerdo; DDVD: disfunção diastólica do ventrículo direito; CVF: capacidade vital forçada; Pulso de O²: pulso de oxigênio; VM: ventilação minuto; RTR: razão de troca respiratória; V'O2: consumo de oxigênio; TCPE: parâmetros do teste cardiopulmonar de exercício. Inclinação VM/VCO²: eficiência ventilatória; $" p<0,05$ entre pacientes com e sem DDVE; $\cdot-p<0,05$ entre pacientes com e sem DDVD.

Nenhum dos parâmetros TCPE, no entanto, se correlaciona com a razão de estresse E/e' (nem no VE nem no VD). Esses achados são semelhantes aos relatados na população em geral. Nedeljkovic et al. detectaram menor carga, menor consumo de oxigênio e menor eficiência ventilatória em pacientes hipertensos com dispneia aos esforços e DDVE induzida por estresse. ${ }^{18}$ Kaiser et al. descreveram aumento da reserva de frequência cardíaca e redução do pulso de oxigênio em uma população geral de pacientes com dispneia aos esforços. ${ }^{19}$ Guazzi et al. também estabeleceram uma associação entre disfunção diastólica (razão E/e') e consumo ao pico de oxigênio, eficiência ventilatória e recuperação da frequência cardíaca. ${ }^{25}$ No grupo de pacientes com patologia cardiovascular evidente e ecocardiografia normal em repouso de Guazzi, a eficiência ventilatória se correlacionou melhor com o pico da razão $E / e^{\prime}>15$. A vantagem clínica da relação $\mathrm{VM} / \mathrm{VCO}_{2}$ como melhor preditor da razão por estresse $\mathrm{E} / \mathrm{e}^{\prime}$ também foi confirmada nos pacientes com insuficiência cardíaca diastólica analisados por Nedeljkovic et al. ${ }^{18}$ Kaiser et al. não apoiam tais conclusões, enfatizando a importância do aumento da reserva da frequência cardíaca e da diminuição no pulso de oxigênio como preditores da razão por estresse E/e' na população geral de pacientes com dispneia aos esforços e livres de doença cardiovascular evidente. ${ }^{19}$
Parece que os parâmetros TCPE podem ajudar no diagnóstico diferencial de dispneia na população em geral, bem como em pacientes com patologia cardiovascular diagnosticada. ${ }^{6-9}$ De acordo com nossos resultados, em pacientes com DPOC, esses não são parâmetros clínicos confiáveis que podem servir como preditores independentes de anormalidade cardiovascular e, portanto, não são aplicáveis no algoritmo de diagnóstico de disfunção diastólica mascarada.

Nossos achados corroboram a presença de comprometimento funcional em pacientes com DPOC não grave com dispneia aos esforços e livres de doença cardiovascular evidente. O Doppler tecidual durante o exercício mostra a complexa interação coração-pulmão e as alterações induzidas pelo esforço, que aumentam os comprometimentos funcionais cardíacos que podem não ser evidentes em repouso. Nossos achados apoiam as recomendações atuais de EES-TCPE como uma ferramenta para detecção precoce de ICFEP. ${ }^{6}$ Como nenhum dos parâmetros do teste de esforço cardiopulmonar provou ser preditivo de DDVE/DDVD induzida por estresse, a ecocardiografia de estresse tem grande relevância clínica para um diagnóstico preciso de patologia cardíaca e respiratória em pacientes com DPOC não graves e dispneia aos esforços. 
Tabela 2 - Parâmetros ecocardiográficos de pacientes com e sem DDVE e DDVD

\begin{tabular}{|c|c|c|c|c|}
\hline & $\begin{array}{c}\text { Pacientes sem DDVE } \\
\text { por estresse (37) }\end{array}$ & $\begin{array}{l}\text { Pacientes com DDVE } \\
\text { por estresse (67) }\end{array}$ & $\begin{array}{l}\text { Pacientes sem DDVD } \\
\text { por estresse (22) }\end{array}$ & $\begin{array}{l}\text { Pacientes com DDVD } \\
\text { por estresse (82) }\end{array}$ \\
\hline \multicolumn{5}{|c|}{ Parâmetros estruturais do VE } \\
\hline FEVE, \%, Simpson & $63,50(60-66)$ & $60,00(57-65)^{*}$ & $65,00(60-66)$ & $61,00(67-65)^{*}$ \\
\hline Septo, $\mathrm{mm}$ & $12,00(11-13)$ & $12,00(11-13)^{*}$ & $12,00(11-12,75)$ & $12,00(11-13)^{*}$ \\
\hline PW, mm & $12,00(11,75-12)$ & $12,00(11-13)^{*}$ & $12,00(11,25-12,75)$ & $12,00(11-13)^{*}$ \\
\hline \multicolumn{5}{|c|}{ Parâmetros funcionais do VE em repouso } \\
\hline Razão E/A & $0,79(0,75-0,85)$ & $0,85(0,76-1,20)^{*}$ & $0,78(0,76-0,83)$ & $0,84(0,75-1,21,)^{*}$ \\
\hline Razão média E/e' & $6,66(6,25-8,33)$ & $6,97(5,76-8,15)^{*}$ & $6,96(6,27-8,33)$ & $6,66(5,63-8,1)^{*}$ \\
\hline \multicolumn{5}{|c|}{ Parâmetros funcionais do VE após o teste de esforço } \\
\hline Razão E/A & $1,25(0,8-1,5)$ & $\neq 1,73(1,55-2,00)^{*}$ & $1,22(0,88-1,37)$ & $\neq \neq 1,71(1,5-2,00)^{*}$ \\
\hline Razão média E/e' & $8,07(6,7-9,6)$ & $\neq 17,33(15,71-8,46)^{*}$ & $8,12(7,25-10)$ & $\begin{array}{c}\neq \neq 17,14(14,66- \\
18,39)^{*}\end{array}$ \\
\hline \multicolumn{5}{|c|}{ Parâmetros estruturais do VD } \\
\hline IVAD, $\mathrm{ml} / \mathrm{m}^{2}$ & $17,57(16,07-19,97)$ & $\neq 22,66(21,31-24,13)^{*}$ & $16,55(15,81-17,54)$ & $\begin{array}{c}\neq \neq 22,27(20,65- \\
23,85)^{*}\end{array}$ \\
\hline RWT, mm & $5,00(4,5-6,5)$ & $\neq 6,50(6-7)^{\star}$ & $5,00(4,12-5,00)$ & $\neq \neq 6,50(6,00-7,00)^{*}$ \\
\hline TAPSE, mm & $23,00(22,00-26,00)$ & $22,00(21,00-23,00)^{*}$ & $23,00(21,25-26,00)$ & $22,00(21-23,5)^{*}$ \\
\hline \multicolumn{5}{|c|}{ Parâmetros funcionais do $\mathrm{VD}$ em repouso } \\
\hline Razão E/A & $0,83(0,75-0,95)$ & $0,69(0,62-0,75)^{*}$ & $0,83(0,76-1,16)$ & $0,71(0,66-0,83)^{*}$ \\
\hline Razão média E/e' & $5,47(4,56-5,69)$ & $4,16(3,33-5,00)^{*}$ & $5,47(4,56-5,69)$ & $4,54(3,33-5,22)^{*}$ \\
\hline TA, msec & $170(163,75-180)$ & $170(160-180)^{*}$ & $170(165-180)$ & $170(160-180)^{*}$ \\
\hline sPAP, mmHg & $26,00(25-28)$ & $28,00(25-30)^{*}$ & $25,00(23-27)$ & $28,00(25-30)^{*}$ \\
\hline \multicolumn{5}{|c|}{ Parâmetros funcionais do VD após o teste de esforço } \\
\hline Razão E/A & $1,26(1,09-1,48)$ & $1,31(1,18-1,49)^{*}$ & $1,28(1,14-1,5)$ & $1,37(1,22-1,52)^{*}$ \\
\hline Razão média E/e' & $6,21(5,38-7,89)$ & $10,83(9,04-13,23)^{*}$ & $6,92(5,46-8,00)$ & $\neq \neq 11,25(9,00-13,33)^{*}$ \\
\hline TA, msec & $165(155-175)$ & \#105(95-110)* & $162,5(155-170)$ & $\neq \neq 110(95-115)^{*}$ \\
\hline sPAP, mmHg & $32,00(30-33,25)$ & $\neq 38,00(36-42)^{*}$ & $32,00(30-33,75)$ & $\neq \neq 38,00(35-40)^{*}$ \\
\hline
\end{tabular}

DDVE: disfunção diastólica do ventrículo esquerdo; DDVD: disfunção diastólica do ventrículo direito; FEVE: fração de ejeção do ventrículo esquerdo; IVAD: índice de volume do átrio direito; TAPSE: excursão sistólica do plano do anel tricúspide; $\neq p<0,05$ entre pacientes com e sem DDVE; PW: parede posterior; SPAP: pressão arterial pulmonar sistólica; EPVD: espessura da parede do ventrículo direito; TA: tempo de aceleração. $\neq \neq p<0,05$ entre pacientes com e sem DDVD.

Tabela 3 - Análise de correlação entre os parâmetros de teste de exercício respiratório e cardiopulmonar com a razão E/e' induzida por estresse para ventrículo esquerdo/ventrículo direito, respectivamente

\begin{tabular}{|c|c|c|c|c|}
\hline \multirow{2}{*}{ Parâmetros } & \multicolumn{2}{|c|}{ DDVE } & \multicolumn{2}{|c|}{ DDVD } \\
\hline & Spearman rho & valor de $p$ & Spearman rho & valor de $p$ \\
\hline Pico de carga, W & 0,02 & 0,84 & 0,03 & 0,78 \\
\hline Pico de VM, I/min & 0,02 & 0,85 & 0,12 & 0,28 \\
\hline Pico de $\mathrm{VO}_{2}, \mathrm{ml} / \mathrm{min} / \mathrm{kg}$ & 0,12 & 0,56 & 0,03 & 0,73 \\
\hline RTR & 0,06 & 0,74 & 0,12 & 0,27 \\
\hline Pico de pulso de $\mathrm{O}_{2} \mathrm{ml} / \mathrm{min} / \mathrm{kg}$ & 0,10 & 0,60 & 0,11 & 0,32 \\
\hline Pico de inclinação $\mathrm{VM} / \mathrm{VCO}_{2}$ & 0,35 & 0,07 & 0,02 & 0,80 \\
\hline CVF, I/min & 0,28 & 0,11 & 0,10 & 0,34 \\
\hline $\mathrm{FEV}_{1}, 1 / \mathrm{min}$ & 0,01 & 0,95 & 0,04 & 0,71 \\
\hline
\end{tabular}

Teste U de Mann-Whitney; † Abreviaturas: DDVE: disfunção diastólica do ventrículo esquerdo; DDVD: disfunção diastólica do ventrículo direito; CVF: capacidade vital forçada; $R T R$ : razão de troca respiratória; $\neq p<0,05$ entre pacientes com e sem DDVE; VM $/ V C O_{2}$, eficiência ventilatória; $\neq \neq p<0,05$ entre pacientes com e sem DDVD 


\section{Limitações do estudo}

O estudo teve as seguintes limitações: 1) tamanho de amostra relativamente pequeno; 2) falta de pletismografia corporal e medida da capacidade de difusão, que são informativos para uma avaliação adequada da dispneia; 3) pacientes com DPOC apresentam oscilações de pressão aumentada durante o ciclo respiratório, e a medição foi realizada ao final da expiração, o que pode influenciar os resultados; 4) as medições foram feitas no início do período de recuperação (aproximadamente dois minutos) após o exercício limitado por sintomas. A linha do tempo das mudanças das pressões pulmonar e intratorácica durante o breve intervalo entre o pico do exercício e sua medida no início da recuperação não é bem conhecida e poderia, portanto, ser subestimada.

\section{Conclusão}

Há uma alta prevalência de disfunção diastólica induzida por estresse em pacientes com DPOC não grave com dispneia aos esforços, sem doença cardiovascular evidente. Nenhum dos parâmetros do TCPE se correlaciona com a razão E/e' induzida por estresse. Portanto, a realização do EES-TCPE é

\section{Referências}

1. Papaioannou A, Bartziokas K, Loukides S, Tsikrika S, Karakontaki F, Haniotou A, et al. Cardiovascular comorbidities in hospitalised DPOC patients: a determinant of future risk? Eur Respir J. 2015;46(3):846-9.

2. Müllerova H, Agusti A, Erqou S, Mapel D. Cardiovascular comorbidity in COPD: systematic literature review. Chest. 2013;144(4):1163-78.

3. Barr RG, Bluemke DA, Ahmed FS, Carr J, Enright L, Hoffman A, et al. Percent emphysema, airflow obstruction, and impaired left ventricular filling. N Engl J Med. 2010;362(3):217-27.

4. Fu M, Zhou J, Thunström E, Almgren T, Grote L, Bollano E, et al. Optimizing the management of heart failure with preserved ejection fraction in the elderly by targeting comorbidities (OPTIMIZE-HFPEF). J Card Fail. 2016;22(7):539-44.

5. Vanfleteren LE, Spruit MA, Groenen M, Gaffron S, Empel V, Bruijnzeel P, et al. Clusters of comorbidities based on validated objective measurements andsystemic inflammation in patients with chronic obstructive pulmonary disease. Am J Respir Crit Care Med. 2013;187(7):728-35.

6. Guazzi M, Arena R, Halle M, Piepoli MF, Myers J, Lavie CJ. Focused update: clinical recommendations for cardiopulmonary exercise testing data assessment in specific patient populations. Circulation. 2016;133(24):694711.

7. Arena R, Sietsema KE. Cardiopulmonary exercise testing in the clinical evaluation of patients with heart and lung disease. Circulation. 2011;123(6):668-80.

8. Arena R, Myers J, Guazzi M. Cardiopulmonary exercise testing is a core assessment for patients with heart failure. Congest Heart Fail. $2011 ; 17(3): 115-9$.

9. Herdy AH, Ritt LE, Stein R, Araújo C, Milani M, Meneghelo RS, et al. Cardiopulmonary exercise test: background, applicability and interpretation. Arq Bras Cardiol. 2016;107(5):467-81.

10. Little WC, Zile MR, Klein A, Appleton CP, Kitzman DW, Wesley-Farrington DJ, et al. Effect of losartan and hydrochlorothiazide on exercise tolerance in the exertional hypertension and left ventricular diastolic dysfunction. Am J Cardiol. 2006;98(3):383-5. necessária para a detecção precoce e o manejo adequado da ICFEP mascarada nesta população.

\section{Contribuição dos autores}

Concepção e desenho da pesquisa, Obtenção de dados, Análise e interpretação dos dados, Análise estatística, Obtenção de financiamento, Redação do manuscrito e Revisão crítica do manuscrito quanto ao conteúdo intelectual importante: Cherneva Z, Cherneva R

\section{Potencial conflito de interesses}

Declaro não haver conflito de interesses pertinentes.

\section{Fontes de financiamento}

O presente estudo não teve fontes de financiamento externas.

\section{Vinculação acadêmica}

Não há vinculação deste estudo a programas de pós-graduação.
11. Miller MR, Hankinson J, Brusasco V, Burgos F, Casaburi R, Coates A, et al. Standardisation of spirometry. Eur Respir J. 2005;26(2):319-38.

12. Mitchell C, Rahko P, Blauwet L, Canaday B, Finstuen JA, Foster MC, et al. Guidelines for performing a comprehensive transthoracic echocardiographic examination in adults: recommendations from the American Society of Echocardiography. J Am Soc Echocardiogr. 2019;32(1):1-64.

13. Nagueh SF, Smiseth OA, Appleton CP, Byrd 3rd BF, Dokainish H, Edvardsen $\mathrm{T}$, et al. Recommendations for the Evaluation of left ventricular diastolic function by echocardiography: an update from the American Society of Echocardiography and the European Association of Cardiovascular Imaging. J Am Soc Echocardiogr. 2016;29(4):277-314.

14. Rudski LG, Lai WW, Afilalo J, Hua L, Handschumacher MD, Chandrasekaran K, et al. Guidelines for the echocardiographic assessment of the right heart in adults: a report from the American Society of Echocardiography endorsed by the European Association of Echocardiography, a registered branch of the European Society of Cardiology, and the Canadian Society of Echocardiography. J Am Soc Echocardiogr. 2010;23(7):685-713.

15. Huang YS, Feng YC, Zhang J, Bai L, Huang W, Li M, et al. Impact of chronic obstructive pulmonary diseases on left ventricular diastolic function in hospitalized elderly patients. Clin Interv Aging. 2014 Dec 19;10:81-7.

16. Kubota Y, Asai K, Murai K, Tsukada YT, Hayashi H, Saito Y, et al. COPD advances in left ventricular diastolic dysfunction. Int J Chron Obstruct Pulmon Dis. 2016 Mar 29;11:649-55.

17. Caram LM, Ferrari R, Naves CR, Tanni SE, Coelho LS, Zanati SG, et al. Association between left ventricular diastolic dysfunction and severity of chronic obstructive pulmonary disease. Clinics. 2013;68(6):772-77.

18. Nedeljkovic I, Banovic M, Stepanovic J, Giga V, Djordjevic-Dikic A, Trifunovic $\mathrm{D}$, et al. The combined exercise stress echocardiography and cardiopulmonary exercise test for identification of masked heart failure with preserved ejection fraction in patients with hypertension. Eur J Prev Cardiol. 2016;23(1):71-7.

19. Kaiser T, Datta D. Can Diastolic dysfunction be identified on cardiopulmonary exercise testing. Chest. 2017;152(4):A976. 
20. Lucas-Ramos P, Izquierdo-Alonso JL, Rodriguez-Gonzalez Moro JM, Frances JF, Lozano PV, Bellón-Cano JM, et al. Chronic obstructive pulmonary disease as a cardiovascular risk factor. Results of a case-control study (CONSISTE study). Int J Chron Obstruct Pulmon Dis. 2012;7:679-86.

21. Fisk M, McEniery CM, Gale N, Mäki-Petäjä K, Forman JR, Munnery M, et al. Surrogate markers of cardiovascular risk and chronic obstructive pulmonary disease: a large case-controlled study. Hypertension. 2018;71(3):499-506.

22. Pelà G, Calzi M, Pinelli S, Roberta Andreoli, Nicola Sverzellati, Giuseppina Bertorelli, et al. Left ventricular structure and remodeling in patients with COPD. Int J Chron Obstruct Pulmon Dis. 2016 May 13;11:1015-22.
23. Neilan TG, Bakker JP, Sharma B, Owens R, Farhad H, Shah R, et al. T1 measurements for detection of expansion of the myocardial extracellular volume in chronic obstructive pulmonary disease. Can J Cardiol. 2014;30(12):1668-75.

24. Sabit R, Bolton CE, Fraser AG, Edwards JM, Edwards PH, Ionescu AA, et al. Sub-clinical left and right ventricular dysfunction in patients with COPD. Respir Med. 2010;104(8):1171-78.

25. Guazzi M, Myers J, Arena R. Cardiopulmonary exercise testing in the clinical and prognostic assessment of diastolic heart failure. J Am Coll Cardiol. 2005;46(10):1883-90. 